\title{
Approximate solutions of cohomological equations associated with some Anosov flows
}

\author{
SVETLANA KATOK† \\ Department of Mathematics, University of California, Santa Cruz, \\ Santa Cruz, CA 95064, USA \\ (Received 3 May 1988)
}

Abstract. The Livshitz theorem reported in 1971 asserts that any $C^{1}$ function having zero integrals over all periodic orbits of a topologically transitive Anosov flow is a derivative of another $C^{1}$ function in the direction of the flow. Similar results for functions of higher differentiability have also appeared since. In this paper we prove a 'finite version' of the Livshitz theorem for a certain class of Anosov flows on 3-dimensional manifolds which include geodesic flows on negatively curved surfaces as a special case.

1. Notations and statement of the main result

Let $X$ be a compact 3-manifold. A flow $\left\{\psi^{t}\right\}(t \in \mathbb{R})$ on $X$ is called contact if it preserves a contact form $\Omega$, i.e. a differential 1 -form such that $\Omega \wedge d \Omega \neq 0$. In this paper we will be concerned with $C^{\infty}$ contact Anosov flows. A primary example of such a flow is a geodesic flow on SM, the unit tangent bundle to a compact surface $M$ provided with a Riemannian metric of negative curvature. We shall introduce some notations and list basic facts about contact Anosov flows.

F1. A flow $\left\{\psi^{t}\right\}$ is Anosov, if there exists a continuous $D \psi^{t}$-invariant splitting of the tangent bundle to $X$

$$
T X=E^{0} \oplus E^{s} \oplus E^{u},
$$

where $E^{0}, E^{s}$ and $E^{u}$ are one-dimensional distributions spanned by unit vector fields $\xi^{0}, \xi^{s}$ and $\xi^{u}$, and for any Riemannian metric there exist constants $a_{1}, b_{1}$, $\alpha>0$ such that for all $x \in X$ and any positive real number $t$

$$
\begin{aligned}
\left\|D \psi^{t} \xi^{s}(x)\right\| & \leq a_{1} e^{-\alpha t}, \\
\left\|D \psi^{t} \xi^{u}(x)\right\| & \geq b_{1} e^{\alpha t} .
\end{aligned}
$$

Here $D$ denotes the differential of the flow, and the norm of a tangent vector is defined by the Riemannian metric on $X$. We shall also need the estimates on the other side which hold for any smooth flow: there exist constants $a_{2}, b_{2}, \delta>0$ such that for all $x \in X$ and any positive real number $t$

$$
\begin{aligned}
\left\|D \psi^{t} \xi^{s}(x)\right\| & \geq a_{2} e^{-\delta t}, \\
\left\|D \psi^{\prime} \xi^{u}(x)\right\| & \leq b_{2} e^{\delta t} .
\end{aligned}
$$

† Supported in part by NSF Postdoctoral Fellowship. 
Let us denote

$$
\chi^{s}(x, t)=\left\|D \psi^{t} \xi^{s}(x)\right\| ; \quad \chi^{u}(x, t)=\left\|D \psi^{i} \xi^{u}(x)\right\| .
$$

Let $-\lambda(x, t)$ and $\mu(x, t)$ be the logarithmic derivatives of the functions $\chi^{s}(x, t)$ and $\chi^{u}(x, t)$, i.e.

$$
\begin{aligned}
& \frac{\partial \chi^{s}(x, t)}{\partial t}=-\lambda(x, t) \chi^{s}(x, t) \\
& \frac{\partial \chi^{u}(x, t)}{\partial t}=\mu(x, t) \chi^{u}(x, t) .
\end{aligned}
$$

We have

$$
\begin{aligned}
& \lambda(x, t)=-\lim _{\tau \rightarrow 0} \frac{\left\|D \psi^{\tau} \xi^{s}\left(\psi^{t} x\right)\right\|-1}{\tau}=\Lambda\left(\psi^{t} x\right), \\
& \mu(x, t)=\lim _{\tau \rightarrow 0} \frac{\left\|D \psi^{\tau} \xi^{u}\left(\psi^{t} x\right)\right\|-1}{\tau}=M\left(\psi^{t} x\right) .
\end{aligned}
$$

The integral curves of $E^{0}$ are the orbits of the flow $\left\{\psi^{t}\right\}$. The integral curves of the distribution $E^{s}\left(E^{u}\right)$ form the stable (unstable) foliation which is denoted by $\sigma^{s}\left(\sigma^{u}\right)$. We denote the distance on $X$ by $d$, and the distance along the leaves of the foliations $\sigma^{s}$ and $\sigma^{u}$ by $d^{s}$ and $d^{u}$ respectively.

F2. A contact Anosov flow $\left\{\psi^{t}\right\}$ preserves the measure on $X$ defined by the volume element $\Omega \wedge d \Omega$, which is sometimes called the Liouville measure. We assume that a Riemannian metric on $X$ is chosen in such a way that the Riemannian volume on $X$ coincides with the Liouville measure.

F3. A contact Anosov flow $\left\{\psi^{t}\right\}$ is topologically transitive, and each leaf of the foliations $\sigma^{s}$ and $\sigma^{u}$ is uniformly dense, i.e. for any $\rho>0$ there exists $N>0$ such that for any $x \in X$, any $M \geq N$ and $i \in\{s, u\} D_{M}^{i}(x)=\left\{z \in \sigma^{i}(x) \mid d^{i}(x, z)<M\right\}$ is $\rho$-dense in $X$, i.e. intersects every ball in $X$ of radius $\rho$ [1].

F4. The distributions $E^{s}$ and $E^{u}$ (and therefore foliations $\sigma^{s}$ and $\sigma^{u}$ ) are of class $C^{2-\varepsilon}$ for any $\varepsilon>0$ [6]. In fact, we only use that they are $C^{1}$. The latter fact for geodesic flows was known already to Hopf $[4, \S 14],[5, \S 7]$. It follows that $\Lambda(x)$, $M(x) \in C^{1}(X)$.

We denote the operators of differentiation in the directions of $\xi^{0}, \xi^{s}$ and $\xi^{u}$ by $\mathscr{D}=\mathscr{D}_{0}, \mathscr{D}_{s}$ and $\mathscr{D}_{u}$ respectively. Let $\alpha^{0}, \alpha^{s}$ and $\alpha^{u}$ be differential 1-forms dual to the vector fields $\xi^{0}, \xi^{s}$ and $\xi^{u}$ :

$$
\alpha^{i}\left(\xi^{j}\right)=\delta_{i j}, \quad \text { for } i, j \in\{0, s, u\}
$$

Hereafter $C$ and $K$ with various subscripts will denote positive constants which may depend on the manifold $X$. The dependence on a parameter, if any, will be specified.

THeorem 1.1. (Finite Livshitz Theorem.) Let $X$ be a compact 3-manifold, $\left\{\psi^{t}\right\}$ be a contact Anosov flow on $X$, and $T>0$. Then for any $\lambda, 0<\lambda<\alpha / \delta$ there exists $a$ constant $C(\lambda)$ such that if $f \in C^{2}(X),\|f\|_{C^{2}}=1$, and $\int_{[0]} f d t=0$ for all periodic orbits $[o]$ of $\left\{\psi^{\prime}\right\}$ of length $\leq T$, then there exist $F, h \in C^{1+\lambda}(X)$ such that $f=\mathscr{D} F+h$, and $\|h\|_{C^{1}} \leq C(\lambda) T^{-\lambda /(3-\lambda)}$. 
Remarks. 1. Notice that a weak form of Theorem 1.1 (without an explicit estimate of how $\|h\|_{C^{1}}$ tends to 0 as $T \rightarrow \infty$ ) follows immediately from the Livshitz† theorem [7] and the fact that the unit sphere in $C^{2}$ is compact in $C^{1}$ (the Ascoli-Arzelà Theorem).

2. For results similar to the Livshitz theorem for functions of higher differentiability see [3], [8] and [6].

\section{Construction of a Hölder continuous differential form on $X$}

Let us fix a Riemannian metric on $X$ as in (F2), and define the following functions $k^{0}(x)=f(x), k^{s}(x)=-\int_{0}^{\infty} \mathscr{D}_{s} f\left(\psi^{t} x\right) \chi^{s}(x, t) d t, k^{u}(x)=-\int_{0}^{-\infty} \mathscr{D}_{u} f\left(\psi^{t} x\right) \chi^{u}(x, t) d t$.

It follows from (1.1) and (1.2) that these integrals converge. We define a differential 1-form $\omega_{f}=\omega$ associated to the function $f$ by the formula $\omega=\omega^{0}+\omega^{s}+\omega^{u}$, where $\omega^{0}=k^{0}(x) \alpha^{0}, \omega^{s}=k^{s}(x) \alpha^{s}, \omega^{u}=k^{u}(x) \alpha^{u}$. For notational simplicity in most cases we will suppress the dependence $\omega_{f}$ on $f$. The following theorem holds for all contact Anosov flows.

THEOREM 2.1. The differential form $\omega_{f}$ satisfies a Hölder condition of order $\lambda$ for any $\lambda, 0<\lambda<1$.

Proof. In view of (F4), it is sufficient to prove that each form $\omega^{0}, \omega^{s}$ and $\omega^{u}$ satisfy a Hölder condition, i.e. that for any $\lambda, 0<\lambda<1$, there exists $C_{0}(\lambda)>0$ such that for $i, j \in\{0, s, u\}$ and $x^{\prime} \in \sigma^{j}(x)\left|k^{i}(x)-k^{i}\left(x^{\prime}\right)\right| \leq C_{0}(\lambda) d^{j}\left(x, x^{\prime}\right)^{\lambda}$. We shall make calculations for $i=s$ and leave the other cases to the reader. Let $d^{j}\left(x, x^{\prime}\right)=d$. If $j=0, s$ we choose $T=T(x)$ such that

$$
\chi^{s}(x, T(x))=d
$$

If $j=u$ we choose $T=T(x)$ such that

$$
d^{u}\left(\psi^{T} x, \psi^{T} x^{\prime}\right)=1
$$

Let us parametrize the piece of the leaf $\sigma^{\prime \prime}(x)$ between $x$ and $x^{\prime}$ by a parameter $u$ as follows: $u(x)=0, u\left(x^{\prime \prime}\right)=d^{u}\left(x, x^{\prime \prime}\right), x^{\prime \prime} \in\left[x, x^{\prime}\right], x^{\prime \prime} \in \sigma^{u}(x)$, and let $l(u, t)=$ $d^{\prime \prime}\left(\psi^{t} x, \psi^{t} x^{\prime \prime}\right)$.

It follows from (1.1) and (1.2) that

$$
a_{2} e^{-\delta(T-t)} \leq d^{u}\left(\psi^{t} x, \psi^{t} x^{\prime \prime}\right) \leq a_{1} e^{-\alpha(T-t)} .
$$

It follows from (1.3) that for any $x^{\prime \prime} \in\left[x, x^{\prime}\right]$

Therefore

$$
\chi^{s}\left(x^{\prime \prime}, t\right)=\exp \int_{0}^{t}-\lambda\left(x^{\prime \prime}, \tau\right) d \tau
$$

$$
\frac{\chi^{s}\left(x^{\prime \prime}, t\right)}{\chi^{s}(x, t)}=\exp \int_{0}^{t}\left(\lambda(x, \tau)-\lambda\left(x^{\prime \prime}, \tau\right)\right) d \tau \leq \exp C_{1} \int_{0}^{t} d^{u}\left(\psi^{\tau} x, \psi^{\tau} x^{\prime \prime}\right) d \tau
$$

For our choice of $T,(2.2)$, we have for $0 \leq t \leq T$ :

$$
\int_{0}^{t} d^{u}\left(\psi^{\tau} x, \psi^{\tau} x^{\prime \prime}\right) d \tau \leq C_{2}
$$

and therefore $\chi^{s}\left(x^{\prime \prime}, t\right) / \chi^{s}(x, t) \leq C_{3}$. The same argument shows that $\dagger$ A phoenetic transliteration of his name, Livcic, appears in the translations of his papers from Russian into English. 
$\chi^{s}(x, t) / \chi^{s}\left(x^{\prime \prime}, t\right) \leq C_{3}$. Hence

$$
C_{3}^{-1} \leq \frac{\chi^{s}\left(x^{\prime \prime}, t\right)}{\chi^{s}(x, t)} \leq C_{3}
$$

for $0 \leq t \leq T$ and any $x^{\prime \prime} \in\left[x, x^{\prime}\right]$. Since the flow $\left\{\psi^{\prime}\right\}$ preserves the Liouville measure (F2), we have $C_{4}^{-1} \leq \chi^{s}\left(x^{\prime \prime}, t\right) \chi^{u}\left(x^{\prime \prime}, t\right) \leq C_{4}$, and therefore

$$
C_{5}^{-1} \leq \frac{\chi^{\prime \prime}\left(x^{\prime \prime}, T\right)}{\chi^{u}(x, T)} \leq C_{5} .
$$

We have $\partial l\left(u\left(x^{\prime \prime}\right), t\right) / \partial u=\left\|D \psi^{t} \xi^{u}\left(x^{\prime \prime}\right)\right\|=\chi^{u}\left(x^{\prime \prime}, t\right)$, and

$$
\begin{aligned}
1= & d^{u}\left(\psi^{T} x, \psi^{T} x^{\prime}\right)=l(d, T) \\
& =\int_{0}^{d} \frac{\partial l}{\partial u}(u, T) d u \leq C_{6} d \chi^{u}(x, T)=C_{7} d\left(\chi^{s}(x, T)^{-1},\right.
\end{aligned}
$$

which implies

$$
\chi^{s}(x, T) \leq C_{7} d, \chi^{s}\left(x^{\prime}, T\right) \leq C_{8} d
$$

We have

$$
\begin{aligned}
\left|k^{s}(x)-k^{s}\left(x^{\prime}\right)\right|= & \mid \int_{0}^{\infty}\left(\mathscr{D}_{s}\left(f\left(\psi^{t} x\right)\right) \chi^{s}(x, t)-\left(\mathscr{D}_{s}\left(f\left(\psi^{\prime} x^{\prime}\right)\right) \chi^{s}\left(x^{\prime}, t\right)\right) d t \mid\right. \\
\leq & \int_{0}^{\infty}\left|\mathscr{D}_{s}\left(f\left(\psi^{t} x\right)\right)-\mathscr{D}_{s}\left(f\left(\psi^{t} x^{\prime}\right)\right)\right| \chi^{s}(x, t) d t \\
& +\int_{0}^{\infty}\left|\mathscr{D}_{s}\left(f\left(\psi^{t} x^{\prime}\right)\right)\right| \cdot\left|\chi^{s}(x, t)-\chi^{s}\left(x^{\prime}, t\right)\right| d t \\
\leq & \int_{0}^{T}\left|\mathscr{D}_{s}\left(f\left(\psi^{\prime} x\right)\right)-\mathscr{D}_{s}\left(f\left(\psi^{t} x^{\prime}\right)\right)\right| \chi^{s}(x, t) d t \\
& +\int_{0}^{T}\left|\mathscr{D}_{s}\left(f\left(\psi^{t} x^{\prime}\right)\right)\right| \cdot\left|\chi^{s}(x, t)-\chi^{s}\left(x^{\prime}, t\right)\right| d t \\
& +\int_{T}^{\infty}\left|\mathscr{D}_{s}\left(f\left(\psi^{t} x\right)\right)-\mathscr{D}_{s}\left(f\left(\psi^{t} x^{\prime}\right)\right)\right| \chi^{s}(x, t) d t \\
& +\int_{T}^{\infty}\left|\mathscr{D}_{s}\left(f\left(\psi^{t} x^{\prime}\right)\right)\right| \cdot\left|\chi^{s}(x, t)-\chi^{s}\left(x^{\prime}, t\right)\right| d t \\
\leq & \int_{0}^{T}\left|\mathscr{D}_{s}\left(f\left(\psi^{t} x\right)\right)-\mathscr{D}_{s}\left(f\left(\psi^{t} x^{\prime}\right)\right)\right| \chi^{s}(x, t) d t \\
& +\int_{0}^{T}\left|\mathscr{D}_{s}\left(f\left(\psi^{t} x^{\prime}\right)\right)\right| \cdot\left|\chi^{s}(x, t)-\chi^{s}\left(x^{\prime}, t\right)\right| d t \\
& +\int_{T}^{\infty}\left|\mathscr{D}_{s}\left(f\left(\psi^{t} x\right)\right)\right| \chi^{s}(x, t) d t+2 \int_{T}^{\infty}\left|\mathscr{D}_{s}\left(f\left(\psi^{t} x^{\prime}\right)\right)\right| \chi^{s}(x, t) d t \\
& +\int_{T}^{\infty}\left|\mathscr{D}_{s}\left(f\left(\psi^{t} x^{\prime}\right)\right)\right| \chi^{s}\left(x^{\prime}, t\right) d t . \\
&
\end{aligned}
$$

We claim that $\chi^{j}\left(x^{\prime}, T\right) \leq C_{9} d$. For $j=u$ this was proved above (2.6). For $j=0, s$ this follows from the choice of $T,(2.1)$, and the inequalities

$$
d^{j}\left(\psi^{t} x, \psi^{t} x^{\prime}\right) \leq C_{10} d
$$


and

$$
\left|\chi^{s}(x, T)-\chi^{s}\left(x^{\prime}, T\right)\right| \leq C_{11} d^{j}\left(\psi^{T} x, \psi^{T} x^{\prime}\right) \leq C_{12} d .
$$

It follows from (1.1) that in both cases

$$
T \leq C_{13}|\ln d| \text {. }
$$

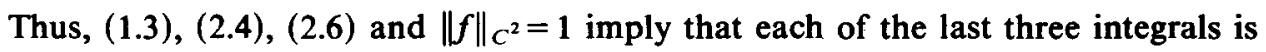
estimated from above by $C_{14} \int_{T}^{\infty} \chi^{s}(x, t) d t \leq C_{15} \chi^{s}(x, T) \leq C_{16} d$.

We estimate now the first two integrals. There exists $\theta(t) \in\left[x, x^{\prime}\right]$ such that

$$
\begin{aligned}
& \int_{0}^{T}\left|\mathscr{D}_{s}\left(f\left(\psi^{t} x\right)\right)-\mathscr{D}_{s}\left(f\left(\psi^{t} x^{\prime}\right)\right)\right| \chi^{s}(x, t) d t \\
& =\int_{0}^{T}\left|\mathscr{D}_{s} \mathscr{D}_{j}\left(f\left(\psi^{t} \theta(t)\right)\right)\right| d^{j}\left(\psi^{t} x, \psi^{t} x^{\prime}\right) \chi^{s}(x, t) d t
\end{aligned}
$$

Let $j=0, s$. The inequalities (1.1) and (1.2) imply that

$$
\int_{0}^{T} \chi^{s}(x, t) d t \leq C_{17}
$$

and using (2.7) we estimate the first integral from above by $C_{18} d$. The second integral in this case is estimated as follows:

$$
\begin{aligned}
\int_{0}^{T}\left|\mathscr{D}_{s}\left(f\left(\psi^{\prime} x^{\prime}\right)\right)\right| \cdot\left|\chi^{s}(x, t)-\chi^{s}\left(x^{\prime}, t\right)\right| d t & \leq C_{19} \int_{0}^{T} d^{j}\left(\psi^{t} x, \psi^{t} x^{\prime}\right) d t \leq C_{20} d T \\
& \leq C_{21} d \cdot|\ln d| \leq C_{21} C_{22}(\lambda) d^{\lambda}
\end{aligned}
$$

for any $\lambda, 0<\lambda<1$. The last two inequalities follow from (2.8) and the fact that for any $\lambda, 0<\lambda<1$ there exists $C_{22}(\lambda)$ such that $d \cdot|\ln d| \leq C_{22}(\lambda) d^{\lambda}$. Now let $j=u$. A calculation similar to $(2.5)$ gives us

$$
d^{u}\left(\psi^{t} x, \psi^{t} x^{\prime}\right)=d \cdot \chi^{u}\left(x_{0}, t\right) \leq C_{4} d \cdot\left(\chi^{s}\left(x_{0}, t\right)\right)^{-1},
$$

where $x_{0} \in\left[x, x^{\prime}\right], x_{0} \in \sigma^{u}(x)$. This equality, together with (2.4) and (2.8), implies that the first integral in this case is estimated from above by

$$
C_{23} \int_{0}^{T}\left|d^{u}\left(\psi^{t} x, \psi^{t} x^{\prime}\right) \chi^{s}(x, t) d t \leq C_{24} d T \leq C_{25} d \cdot\right| \ln d \mid \leq C_{26}(\lambda) d^{\lambda}
$$

for any $\lambda, 0<\lambda<1$. The second integral is estimated from above by $C_{27} \int_{0}^{T} \mid \chi^{s}(x, t)-$ $\chi^{s}\left(x^{\prime}, t\right) \mid d t$. We use (1.3) and (2.3) to estimate the integrand

$$
\begin{aligned}
\left|\chi^{s}(x, t)-\chi^{s}\left(x^{\prime}, t\right)\right| & =\left|\exp \int_{0}^{t}-\lambda(x, \tau) d \tau-\exp \int_{0}^{t}-\lambda\left(x^{\prime}, \tau\right) d \tau\right| \\
& =\left(\exp \int_{0}^{t}-\lambda(x, \tau) d \tau\right) \cdot\left|1-\exp \int_{0}^{t}\left(\lambda(x, \tau)-\lambda\left(x^{\prime}, \tau\right)\right) d \tau\right| \\
& \leq C_{28} \chi^{s}(x, t) \cdot\left|\int_{0}^{t}\left(\lambda(x, \tau)-\lambda\left(x^{\prime}, \tau\right)\right) d \tau\right| \\
& \leq C_{29} \chi^{s}(x, t) \int_{0}^{t} d^{u}\left(\psi^{\tau} x, \psi^{\tau} x^{\prime}\right) d \tau \\
& \leq C_{30} \chi^{s}(x, t) \int_{0}^{t} d \cdot\left(\chi^{s}(x, \tau)\right)^{-1} d \tau \\
& \leq C_{31} \chi^{s}(x, t)\left(\chi^{s}(x, t)\right)^{-1} \cdot d \leq C_{32} d .
\end{aligned}
$$


Thus the second integral is estimated by $C_{32} d T \leq C_{33} d \cdot|\ln d| \leq C_{34}(\lambda) d^{\lambda}$ for any $\lambda, 0<\lambda<1$, and the theorem follows.

3. Construction of an $\varepsilon$-dense orbit for the flow $\left\{\psi^{t}\right\}$

TheOREM 3.1. Given $\varepsilon>0$ sufficiently small, there exists an $\varepsilon$-dense piece of orbit of the flow $\left\{\psi^{t}\right\}$ of length $T$ :

$$
\mathcal{O}=\left\{x \in X, x=\psi^{t} x_{0}, 0 \leq t \leq T\right\}
$$

with $T=C \ln \varepsilon^{-1} / \varepsilon^{2}$ where the constant $C>0$ depends only on the Riemannian metric on the manifold $X$ and the flow $\left\{\psi^{t}\right\}$.

Proof. We prove first that any two points $x, y \in X$ can be ' $\varepsilon$-joined' by a piece of orbit of length $C_{1} \ln \varepsilon^{-1}$, i.e. there exist $x^{\prime}, y^{\prime} \in X$ and a constant $C_{1}>0$ such that $d\left(x, x^{\prime}\right)<\varepsilon, d\left(y, y^{\prime}\right)<\varepsilon$, and $y^{\prime}=\psi^{T} x^{\prime}$ for $T=C_{1} \ln \varepsilon^{-1}$. Let $\rho$ be a sufficiently small number which will be specified below. We assume that $\varepsilon<\rho$. By (F3) one can choose a constant $N>0$ such that any piece of the leaf of the foliation $\sigma^{u}$ of size $M \geq N$ is $\rho / 2$-dense. For $T_{1}=\alpha^{-1} \ln \left(N / b_{1} \varepsilon\right)$ the piece of the leaf $\psi^{T_{1}}\left(D_{\varepsilon}^{u} y\right)=$ $D_{M}^{u}\left(\psi^{T_{1}} y\right)$ is of size $M>N$, and therefore is $\rho / 2$-dense. Let $T_{0}=2 \alpha^{-1} \ln \left(a_{1} \rho / \varepsilon\right)$, and $x_{0}=\psi^{-T_{0}} x$. For small enough $\rho$ there exist $y_{0} \in D_{M}^{u}\left(\psi^{\left.T_{1} y\right)}\right.$ and $z=\psi^{t} y_{0}$ with $|t|<\rho$ such that $z \in D_{\rho}^{s}\left(x_{0}\right)$. We also have $d\left(\psi^{T_{0}} z, \psi^{T_{0}} x_{0}\right)=d\left(\psi^{T_{0}} z, x\right) \leq a_{1} \rho e^{-\alpha T_{0}}<\varepsilon$. Thus we obtained two points $x^{\prime}=\psi^{T_{0}}$ and $y^{\prime}=\psi^{-T_{1}} y_{0}$ such that $d\left(x, x^{\prime}\right)<\varepsilon$, $d\left(y, y^{\prime}\right)<\varepsilon$, and $y^{\prime}=\psi^{T} x^{\prime}$. If $\varepsilon<\min \left(1 / a_{1} \rho, b_{1} / N, e^{-\rho}, \rho\right)$ then for some constant $C_{1}>0 T=T_{0}+T_{1}+d\left(y_{0}, z\right)<C_{1} \ln \varepsilon^{-1}$.

For each point $x_{0} \in X$ we define the following cylinder sets:

$$
C_{\rho}\left(x_{0}\right)=\left\{x=(z, t) \mid z \in S_{\rho}\left(x_{0}\right),-\rho<t<\rho\right\}
$$

where $S_{\rho}\left(x_{0}\right)$ is a 2-dimensional smooth local cross-section transversal to the flow $\left\{\psi^{\prime}\right\}$ passing through $x_{0}$, and $x=\psi^{t} z$. To complete the proof of the theorem we choose a cover of $X$ by a finite number of cylinders $C_{\rho}\left(x_{i}\right), i=0,1,2, \ldots, N$. Let us choose a smooth coordinate system in each local cross-section $S_{\rho}\left(x_{i}\right)$. Then it makes sense to talk about square lattices in $S_{\rho}\left(x_{i}\right)$ relative to this coordinate system.

Definition. We call a set of points in $S_{p}\left(x_{i}\right) \varepsilon$-regular if it is an $\varepsilon^{2}$-perturbation of some square lattice in $S_{\rho}\left(x_{i}\right)$ of size $\varepsilon / 2$.

For each $i=0,1,2, \ldots, N$ we choose an $\varepsilon$-regular set $E_{i} \subset S_{\rho}\left(x_{i}\right)$, and let $\Lambda_{i}=$ $\left\{x \in C_{\rho}\left(x_{i}\right) \mid z \in E_{i}\right\}$. The number of pieces in $\bigcup_{i=0}^{N} \Lambda_{i}$ is $C_{2} / \varepsilon^{2}$. We can ' $\varepsilon^{2}$-join' them together using the estimate in the beginning of the proof. An application of the Bowen specification theorem [2] gives us a desired $\varepsilon$-dense piece of orbit of length $C \ln \varepsilon^{-1} / \varepsilon^{2}$ which we denote by $O$.

Remark. By the Bowen specification theorem [2] for each $i=0,1, \ldots, N, O$ contains a subset $\bar{\Lambda}_{i}$ consisting of a number of pieces approximating pieces of orbits constituting $\Lambda_{i}$. Let $\mathscr{S}=\bigcup_{i=0}^{N} \bar{\Lambda}_{i}$. Notice that $\mathscr{S} \cap S_{\rho}\left(x_{i}\right)$ is also an $\varepsilon$-regular set for each $i=0, \ldots, N$.

\section{The proof of Theorem 1.1}

Definition. Let $r$ be the injectivity radius of $X$. We say that a function $F$ defined on $\mathscr{E}$, a subset of $X$, is of class $C_{K}^{1+\lambda}(0<\lambda<1, K>0)$ if there exists a family of linear functions $l_{x}(v)$ for $x \in X, v \in T_{x} X$ such that for any $x, y \in \mathscr{E}, d(x, y)<r$,

$$
\left|F(y)-F(x)-l_{x}\left(v_{x y}\right)\right| \leq K d(x, y)^{1+\lambda},
$$


where $v_{x y} \in T_{x} X$ is a tangent vector to the geodesic from $x$ to $y$ (on $X$ ) of length $d(x, y)$.

LEMMA 4.1. Let $O$ be a piece of orbit of the flow $\left\{\psi^{t}\right\}$ of length $T$, and $f \in C^{2}(X)$ is such that $\|f\|_{C^{2}}=1$ and $\int_{[o]} f d t=0$ for all periodic orbits $[0]$ of $\left\{\psi^{t}\right\}$ of length $\leq T$. We define the following function on $O$ : for $x=\psi^{t} x_{0}, 0 \leq t \leq T$

$$
F(x)=\int_{0}^{t} f\left(\psi^{s} x_{0}\right) d s
$$

Then for any $\lambda, 0<\lambda<\alpha / \delta$ there exists $K_{0}(\lambda)$ such that $F(x)$ is of class $C_{K_{0}(\lambda)}^{1+\lambda}$ on $O$. Proof. We shall show that the role of the linear function $l_{x}$ is played by the differential form $\omega_{f}$ introduced in $\S 2$. For $j=s$, $u$ we define $\sigma^{0, j}(x)=\left\{y=\psi^{t} z,-\infty<t<\infty\right.$, for some $\left.z \in \sigma^{j}(x)\right\}$; they are called leaves of the weak stable (for $j=s$ ) and the weak unstable (for $j=u$ ) foliations. Let $d^{0, j}$ denote the distance on $\sigma^{0, j}, \rho$ be as in $\S 3$, and $D_{p}^{0, j}(x)=\left\{y \in \sigma^{0, j}(x) \mid d^{0, j}(x, y)<\rho\right\}$. Suppose $x_{0}=x, y_{0}=y \in O, y_{0}=\psi^{T^{\prime}} x_{0}, T^{\prime} \leq$ $T, d\left(x_{0}, y_{0}\right)=d<r$. Let $z_{0}=z=D_{\rho}^{s}\left(x_{0}\right) \cap D_{\rho}^{0, u}\left(y_{0}\right)$, and $y_{00}=\psi^{T_{0}} x_{0},\left|T_{0}-T^{\prime}\right|<d$, $y_{00} \in D_{\rho}^{u}\left(z_{0}\right)$. We denote the arc of $\sigma^{s}(x)$ between $x$ and $z$ by $\sigma^{s}(x, z)$, the arc of $\sigma^{u}\left(y_{00}\right)$ between $y_{00}$ and $z$ by $\sigma^{u}\left(y_{00}, z\right)$, and the piece of orbit $\sigma$ between $y$ and $y_{00}$ by $O\left(y, y_{00}\right)$. Notice that

$$
\begin{aligned}
\int_{\sigma^{s}(x, z)} \omega_{f} & =\int_{0}^{\infty}\left(f\left(\psi^{\prime} x\right)-f\left(\psi^{\prime} z\right)\right) d t, \\
\int_{\sigma^{\prime \prime}\left(y_{00} z\right)} \omega_{f} & =\int_{0}^{-\infty}\left(f\left(\psi^{t} y_{00}\right)-f\left(\psi^{t} z\right)\right) d t, \\
\int_{O\left(y, y_{00}\right)} \omega_{f} & =\int_{0}^{T_{0}-T} f\left(\psi^{t} y\right) d t .
\end{aligned}
$$

The fact that $\omega_{f}$ satisfies a Hölder condition of order $\lambda$ (Theorem 2.1) and (4.2) imply that Lemma 4.1 follows from the following statement: given $\lambda, 0<\lambda<\alpha / \delta$, there exists a constant $K(\lambda)$ such that for any $x, y \in \mathcal{O}, y=\psi^{T_{0}} x, T_{0} \leq T, d(x, y)=d<r$ with the property that $D_{\rho}^{s}\left(x_{0}\right) \cap D_{\rho}^{u}\left(y_{0}\right) \neq \varnothing$, there exists a constant $K(\lambda)$ such that for $D_{\rho}^{s}\left(x_{0}\right) \cap D_{\rho}^{u}\left(y_{0}\right)=z$

$$
\begin{aligned}
& \left|(F(y)-F(x))-\left(\int_{0}^{\infty}\left(f\left(\psi^{t} x\right)-f\left(\psi^{t} z\right)\right) d t+\int_{0}^{-\infty}\left(f\left(\psi^{t} z\right)-f\left(\psi^{t} y\right)\right) d t\right)\right| \\
& \leq K(\lambda) d(x, y)^{1+\lambda} .
\end{aligned}
$$

We notice that it is sufficient to prove the above statement for sufficiently small $d$. Without loss of generality we may assume that $x=x_{0}$ and therefore $F(x)=0$. We construct five sequences of points $\left\{x_{j}\right\},\left\{y_{j}\right\},\left\{y_{j j}\right\},\left\{z_{j}\right\}$ and $\left\{u_{j}\right\}$, and a sequence of numbers $\left\{T_{j}\right\}(j=0,1,2, \ldots)$ inductively as follows:

$$
\begin{gathered}
x_{0}=x, \quad y_{0}=y_{00}=y=\psi^{T_{0}} x_{0}, \quad z_{0}=z=D_{\rho}^{s}\left(x_{0}\right) \cap D_{\rho}^{0, u}\left(y_{0}\right), \\
y_{00} \in D_{\rho}^{u}\left(z_{0}\right), \quad u_{0} \in D_{\rho}^{u}\left(x_{0}\right), \quad \psi^{T_{0}} u_{0}=z_{0}, \\
x_{j}=D_{\rho}^{s}\left(u_{j-1}\right) \cap D_{\rho}^{0, u}\left(y_{j-1, j-1}\right)=D_{\rho}^{0, u}\left(z_{j-1}\right), \quad y_{j}=\psi^{T_{j-1}} x_{j}, \\
z_{j}=D_{\rho}^{s}\left(x_{j}\right) \cap D_{\rho}^{0, u}\left(y_{j}\right), \quad y_{j j}=\psi^{T_{j}} x_{j}, \\
y_{j j} \in D_{\rho}^{u}\left(z_{j}\right), \quad u_{j} \in D_{\rho}^{u}\left(x_{j}\right), \quad \psi^{T_{j}} u_{j}=z_{j}
\end{gathered}
$$


Let $x \in X, S_{\rho}(x)$ be as in $\S 3$, and $\varphi: S_{\rho}(x) \rightarrow S_{\rho}(x)$ be a return map for the flow $\left\{\psi^{t}\right\}$. For any $y \in S_{\rho}(x)$ we let $P_{\rho}^{j}(y)=\sigma^{0, j}(y) \cap S_{\rho}(x)$ for $j=s$, $u$. The local foliations $P_{\rho}^{j}$ are stable and unstable foliations for the return map $\varphi$. They inherit the smoothness of the weak foliations $\sigma^{0, j}$ which is not less than the smoothness of foliations $\sigma^{j}$. Let $l^{j}$ be the distance on the leaves of the local foliation $P_{p}^{j}(j=s, u)$. We assume that $\rho$ is chosen such that there exists a constant $C_{1}>1$ such that for any $x, y \in P_{\rho}^{j}$

$$
C_{1}^{-1}<\frac{l^{j}(x, y)}{d(x, y)}<C_{1} .
$$

Thus it follows from (F4) that there exists a constant $C_{2}>1$ such that for any 'quadrangle' $\left[x_{1}, x_{2}, x_{3}, x_{4}\right]$ such that

$$
x_{2} \in P_{\rho}^{u}\left(x_{1}\right), x_{3} \in P_{\rho}^{s}\left(x_{2}\right), x_{4} \in P_{\rho}^{u}\left(x_{3}\right), x_{1} \in P_{\rho}^{s}\left(x_{4}\right),
$$

the following inequalities hold:

$$
C_{2}^{-1} \leq \frac{d\left(x_{1}, x_{2}\right)}{d\left(x_{4}, x_{3}\right)} \leq C_{2}, \quad C_{2}^{-1} \leq \frac{d\left(x_{2}, x_{3}\right)}{d\left(x_{1}, x_{4}\right)} \leq C_{2} .
$$

Let $\pi: C_{\rho}\left(x_{0}\right) \rightarrow S_{\rho}\left(x_{0}\right)$ be defined by the formula $\pi(z, t)=z$, and $S_{j}=$ $T_{0}+\cdots+T_{j-1}$. By properties (1.1) and (1.2), for some constants $C_{3}, C_{4}, C_{5}, C_{6}>0$, and $j=0,1, \ldots$ we have

$$
\begin{aligned}
C_{3} e^{-\delta S_{j}} d & \leq d\left(\pi x_{j}, \pi y_{j j}\right) \leq C_{4} e^{-\alpha S_{j} d}, \\
d\left(\pi x_{j}, x_{0}\right) & <C_{5} d, d\left(\pi y_{j}, x_{0}\right)<C_{5}, \\
\left|T_{j}-T_{0}\right| & \leq C_{6} d .
\end{aligned}
$$

Therefore the sequences $\left\{\pi x_{j}\right\},\left\{\pi y_{j}\right\},\left\{\pi y_{j j}\right\},\left\{\pi z_{j}\right\}$ and $\left\{\pi u_{j}\right\}$ converge in $S_{\rho}\left(x_{0}\right)$ to a fixed point of the continuous map $\varphi: S_{\rho}(x) \rightarrow S_{\rho}(x)$. The orbit of the flow $\left\{\psi^{t}\right\}$ passing through this point is a periodic orbit. This completes a well-known proof of the Anosov closing lemma. For our purposes, however, we have to look closely at the rate of convergence of this process. Let $k$ be an integer, $k \geq 1$ (it will be chosen later, in (4.7)). We have

$$
\begin{gathered}
F(y)=\int_{0}^{T_{0}} f\left(\psi^{t} x\right) d t=\sum_{j=0}^{k-1}\left(\int_{0}^{T_{0}}\left(f\left(\psi^{t} x_{j}\right)-f\left(\psi^{t} z_{j}\right)\right) d t+\int_{0}^{T_{0}}\left(f\left(\psi^{t} z_{j}\right)-f\left(\psi^{t} x_{j+1}\right)\right) d t\right) \\
+\int_{0}^{T_{0}} f\left(\psi^{t} x_{k}\right) d t .
\end{gathered}
$$

On the other hand,

$$
\int_{0}^{\infty}\left(f\left(\psi^{t} x\right)-f\left(\psi^{t} z\right)\right) d t=\sum_{j=0}^{k-1} \int_{S_{j}}^{S_{j+1}}\left(f\left(\psi^{t} x\right)-f\left(\psi^{t} z\right)\right) d t+\int_{S_{k}}^{\infty}\left(f\left(\psi^{t} x\right)-f\left(\psi^{t} z\right)\right) d t
$$

and

$$
\begin{aligned}
\int_{0}^{-\infty}( & \left.f\left(\psi^{t} z\right)-f\left(\psi^{t} y\right)\right) d t \\
& =\sum_{j=0}^{k-1} \int_{-S_{j}}^{-S_{j+1}}\left(f\left(\psi^{t} z\right)-f\left(\psi^{t} y\right)\right) d t+\int_{-S_{k}}^{-\infty}\left(f\left(\psi^{t} z\right)-f\left(\psi^{t} y\right)\right) d t
\end{aligned}
$$


An easy calculation gives us the following estimate:

$$
\begin{aligned}
& \left|F(y)-\left(\int_{0}^{\infty}\left(f\left(\psi^{t} x\right)-f\left(\psi^{t} z\right)\right) d t+\int_{0}^{-\infty}\left(f\left(\psi^{t} z\right)-f\left(\psi^{t} y\right)\right) d t\right)\right| \\
& \quad \leq \Sigma_{1}+\Sigma_{2}+\Sigma_{3}+\Sigma_{4}+I_{1}+I_{2}+I_{3},
\end{aligned}
$$

where

$$
\begin{aligned}
& \Sigma_{1}=\left|\sum_{j=1}^{k-1} \int_{0}^{T_{j}}\left(f\left(\psi^{t} x_{j}\right)-f\left(\psi^{t} z_{j}\right)\right)-\left(f\left(\psi^{S_{j}+t} x\right)-f\left(\psi^{S_{j}+t} z\right)\right) d t\right| \\
& \Sigma_{2}=\left|\sum_{j=0}^{k-1} \int_{0}^{T_{j}}\left(f\left(\psi^{t} z_{j}\right)-f\left(\psi^{t} x_{j+1}\right)\right)-\left(f\left(\psi^{t-S_{j+1}} y\right)-f\left(\psi^{t-S_{j+1} z}\right)\right) d t\right|, \\
& \Sigma_{3}=\left|\sum_{j=0}^{k-1} \int_{T_{0}}^{T_{j}}\left(f\left(\psi^{\prime} x_{j}\right)-f\left(\psi^{t} z_{j}\right)\right) d t\right|, \quad \Sigma_{4}=\left|\sum_{j=0}^{k-1} \int_{T_{0}}^{T_{j}}\left(f\left(\psi^{t} z_{j}\right)-f\left(\psi^{t} x_{j+1}\right)\right) d t\right|, \\
& I_{1}=\left|\int_{0}^{T_{0}} f\left(\psi^{t} x_{k}\right) d t\right|, \quad I_{2}=\left|\int_{S_{k}}^{\infty}\left(f\left(\psi^{t} x\right)-f\left(\psi^{t} z\right)\right) d t\right|, \\
& I_{3}=\left|\int_{-S_{k}}^{-\infty}\left(f\left(\psi^{\prime} z\right)-f\left(\psi^{\prime} y\right)\right) d t\right| \text {. }
\end{aligned}
$$

It follows from (4.3) that for $j \geq 0$

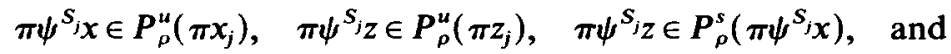

$$
\begin{aligned}
& \pi \psi^{-s_{j+1}} y \in P_{\rho}^{s}\left(\pi z_{j}\right), \quad \pi \psi^{-s_{j+1}} z \in P_{\rho}^{s}\left(\pi x_{j+1}\right), \quad \pi \psi^{-s_{j+1}} y \in P_{\rho}^{u}\left(\pi \psi^{-s_{j+1} z}\right) .
\end{aligned}
$$

Therefore the two 'quadrangles' $Q_{1}(t)=\left[\pi \psi^{\prime} x_{j}, \pi \psi^{t} z_{j}, \pi \psi^{s_{j}+t} z, \pi \psi^{S_{j}+t} x\right]$ and $Q_{2}(t)=$ $\left[\pi \psi^{t} z_{j}, \pi \psi^{t} x_{j+1}, \pi \psi^{t-S_{j+1}} z, \pi \psi^{t-S_{j+1}} y\right]$ satisfy (4.4), and we obtain the following estimates for the lengths of their sides:

$$
\begin{aligned}
d\left(\pi \psi^{t} x_{j}, \pi \psi^{S_{j}+t} x\right) & \leq C_{7} d\left(\pi \psi^{T_{j}} x_{j}, \pi \psi^{S_{j}} \psi^{T_{j}} x\right) \leq C_{8} e^{\delta S_{j}} d \leq C_{8} e^{\delta S_{k-1}} e, \\
d\left(\pi \psi^{t} x_{j}, \pi \psi^{t} z_{j}\right) & \leq C_{9} d\left(\pi x_{j}, \pi z_{j}\right) \leq C_{10} e^{-\alpha S_{j}} d, \\
d\left(\pi \psi^{t} z_{j}, \pi \psi^{t-S_{j+1}} y\right) & \leq C_{11} d\left(\pi z_{j}, \pi \psi^{-S_{j+1}} y\right) \leq C_{12} e^{\delta S_{j}} d \leq C_{12} e^{\delta S_{k-1} d}
\end{aligned}
$$

and

$$
d\left(\pi \psi^{t} z_{j}, \pi \psi^{t} x_{j+1}\right) \leq C_{13} d\left(\pi \psi^{T_{j}} z_{j}, \pi \psi^{T_{j}} x_{j+1}\right) \leq C_{14} d\left(\varphi \pi z_{j}, \varphi \pi x_{j+1}\right) \leq C_{15} e^{-\alpha S_{j}} d .
$$

If $\boldsymbol{d}$ is sufficiently small, we can choose $k \geq 1$ satisfying the following inequalities

$$
\left(\max C_{12}, C_{8}\right)^{-1} \rho e^{-\delta s_{k}} \leq d<e^{-\delta S_{k-1}} \rho\left(\max C_{12}, C_{8}\right)^{-1},
$$

it will follow that $Q_{1}(t), Q_{2}(t) \subset S_{\rho}(x)$, and therefore (4.5) applies. We have $e^{s_{k-1}} \leq$ $C_{16} d^{-1 / \delta}, S_{k-1} \leq C_{17} \ln d^{-1}$. Thus, for some point $\theta(t) \in Q_{1}(t)$ we have

$$
\begin{aligned}
& \left|\sum_{j=1}^{k-1} \int_{0}^{T_{j}}\left(f\left(\pi \psi^{t} x_{j}\right)-f\left(\pi \psi^{t} z_{j}\right)\right)-\left(f\left(\pi \psi^{S_{j}+t} x\right)-f\left(\pi \psi^{S_{j}+t} z\right)\right) d t\right| \\
& \quad \leq C_{18} \sum_{j=1}^{k-1}\left|\int_{0}^{T_{j}} \mathscr{D}_{\mathcal{L}} \mathscr{D}_{s}(f(\theta(t))) \cdot d\left(\pi \psi^{t} x_{j}, \pi \psi^{t} z_{j}\right) \cdot d\left(\pi \psi^{t} x_{j}, \pi \psi^{S_{j}+t} x\right) d t\right| \\
& \quad \leq C_{19}(k-1) T d^{2} e^{S_{k-1}(\delta-\alpha)} \leq C_{20} d^{\alpha / \delta} d \cdot \ln d^{-1} \leq C_{21}(\lambda) d^{1+\lambda} .
\end{aligned}
$$


and therefore $\Sigma_{1} \leq C_{22}(\lambda) d^{1+\lambda}$ for any $\lambda, 0<\lambda<\alpha / \delta$. Similarly we obtain the estimate $\Sigma_{2} \leq C_{23}(\lambda) d^{1+\lambda}$ for any $\lambda, 0<\lambda<\alpha / \delta$.

In order to estimate $I_{1}$ we let

$$
\mathcal{O}\left(x_{k}\right)=\left\{x \in X, x=\psi^{t} x_{k}, 0 \leq t \leq T_{0}\right\} .
$$

By the Anosov closing lemma, using the fact that $d\left(x_{k}, \psi^{T_{k}} x_{k}\right)=d\left(x_{k}, y_{k k}\right) \leq$ $C_{1} e^{-\alpha S_{k}} d$, and that the integral of $f(x)$ over any closed orbit of length $\leq T$ is equal to zero, we obtain the following estimate:

$$
I_{1}=\left|\int_{0}^{T_{0}} f\left(\psi^{t} x_{k}\right) d t\right| \leq C_{24} e^{-\alpha S_{k}} \cdot d \leq C_{25} d^{1+(\alpha / \delta)} .
$$

The last inequality follows from (4.7). Following the previous argument we conclude that

$$
I_{2}=\left|\int_{S_{k}}^{\infty}\left(f\left(\psi^{t} x\right)-f\left(\psi^{t} z\right)\right) d t\right| \leq C_{26} \int_{S_{k}}^{\infty} e^{-\alpha t} d t \cdot d=\frac{C_{27}}{\alpha} e^{-\alpha S_{k}} \cdot d \leq C_{28} d^{1+(\alpha / \delta)}
$$

Similarly,

$$
I_{3}=\left|\int_{-S_{k}}^{-\infty}\left(f\left(\psi^{t} z\right)-f\left(\psi^{t} y\right)\right) d t\right| \leq C_{29}\left|\int_{-S_{k}}^{-\infty} e^{\delta t} d t\right|=\frac{C_{30}}{\delta} e^{-\delta S_{k}} \cdot d \leq C_{31} d^{2} .
$$

Using (4.6) we obtain the following estimates for $\Sigma_{3}$ and $\Sigma_{4}$ :

$$
\begin{aligned}
& \Sigma_{3}=\left|\sum_{j=0}^{k-1} \int_{T_{0}}^{T_{j}}\left(f\left(\psi^{t} x_{j}\right)-f\left(\psi^{t} z_{j}\right)\right) d t\right| \leq C_{32} d \sum_{j=0}^{k-1} d\left(\pi \psi^{T} x_{j}, \pi \psi^{T} z_{j}\right) \leq C_{33} d^{2}, \\
& \Sigma_{4}=\left|\sum_{j=0}^{k-1} \int_{T}^{T_{j}}\left(f\left(\psi^{t} z_{j}\right)-f\left(\psi^{t} x_{j+1}\right)\right) d t\right| \leq C_{34} d^{2} .
\end{aligned}
$$

This concludes the proof of Lemma 4.1.

CoRollary. $F(x)$ is of class $C_{K_{0}(\lambda)}^{1+\lambda}$ on the set $\mathscr{S}$ (cf., Remark in $\S 3$ ), for any $\lambda$, $0<\lambda<\alpha / \delta$.

LEMMA 4.2. For any $\lambda, 0<\lambda<\alpha / \delta$ there exist constants $K_{1}(\lambda), K_{2}(\lambda), K_{3}(\lambda)$ such that the function $F(x)$ can be extended from $\mathscr{S}$ to $X$ as a function of class $C_{K_{1}(\lambda)}^{1+\lambda}$ in such a way that $\mathscr{D} F(x) \in C_{K_{2}(\lambda)}^{1+\lambda}(X)$, and for $h(x)=\mathscr{D} F(x)-f(x)$ we have $\|h(x)\|_{C^{\prime}} \leq$ $K_{3}(\lambda) \varepsilon^{\lambda}$.

Proof. First, we show how to extend $F(x)$ from $\mathscr{S}_{\cap} S_{p}\left(x_{0}\right)$ to $S_{\rho}\left(x_{0}\right)$ as a function of class $C_{K_{1}(\lambda)}^{1+\lambda} . \mathscr{S} \cap S_{\rho}\left(x_{0}\right)$ is a discrete $\varepsilon$-regular set. It is sufficient to extend $F(x)$ to a 'generating' quadrangle of $\mathscr{I}_{\cap} S_{\rho}\left(x_{0}\right)$ which is a $\varepsilon^{2}$-perturbation of a square. We denote its vertices by $A, B, C$ and $D$, and the directions $A \vec{B}$ and $A \vec{D}$ by $x$ and $y$ respectively. We may assume that $F$ is a real-valued function since the following argument is valid for $\operatorname{Re} F$ and $\operatorname{Im} F$. We extend $F(x)$ to the interval $[A, B]$ knowing $F(A), F(B)$ and $F_{x}^{\prime}(A)=l_{A}\left(v_{A B}\right)=a_{A}, F_{x}^{\prime}(B)=-l_{B}\left(v_{B A}\right)=a_{B}$. There exist $t_{A B} \in$ $[A, B]$ and $k_{A B} \in \mathbb{R}$ such that

$$
F_{x}^{\prime}(w)=\left\{\begin{array}{l}
k_{A B} d(w, A)+a_{A}, \quad w \in\left[A, t_{A B}\right] \\
-k_{A B} d(w, A)+k_{A B} d(A, B)+a_{B}, \quad w \in\left[t_{A B}, B\right]
\end{array}\right.
$$


and $\int_{A}^{B} F_{x}^{\prime}(w) d w=F(B)-F(A) . F(x)$ is of class $C_{K_{0}(\lambda)}^{1+\lambda}$. It follows from (4.8) that $k_{A B}$ satisfies the following quadratic equation

$$
d(A, B)^{2} k_{A B}^{2}-2\left[2(F(B)-F(A))-d(A, B)\left(a_{A}+a_{B}\right)\right] k_{A B}-\left(a_{A}-a_{B}\right)^{2}=0 .
$$

A direct calculation shows that

$$
\left|k_{A B}\right| \leq 4 K_{0}(\lambda) d(A, B)^{\lambda-1},
$$

Then for $w, w^{\prime} \in[A, B]$

$$
\left|F_{x}^{\prime}(w)-F_{x}^{\prime}\left(w^{\prime}\right)\right| \leq\left|k_{A B}\right| d\left(w, w^{\prime}\right) \leq 4 K_{0}(\lambda) d\left(w, w^{\prime}\right)^{\lambda} .
$$

We define $F_{y}^{\prime}(w)$ linearly for $w \in[A, B]$ :

$$
F_{y}^{\prime}(w)=\frac{F_{y}^{\prime}(A) d(w, B)+F_{y}^{\prime}(B) d(A, w)}{d(A, B)} .
$$

Then $\left|F_{y}^{\prime}(w)-F_{y}^{\prime}\left(w^{\prime}\right)\right| \leq K_{0}(\lambda) d\left(w, w^{\prime}\right)^{\lambda}$. Thus $F(x)$ is extended to $[A B]$, and analogously to $[B C],[C D]$ and $[D A]$, as a $C_{4 K_{0}(\lambda)}^{1+\lambda}$-function. We parametrize each interval $[A B]$ and $[C D]$ by its normalized length $\sigma$. Then we connect points having the same parameter by an interval of a geodesic, obtaining a family of coordinate curves, and extend $F(x)$ to each interval by the formulas (4.8) and (4.10) as a $C_{C_{1}(\lambda)}^{1+\lambda}$-function for some $C_{1}(\lambda)>0$. Thus we obtain a function inside the quadrangle $A B C D$. In order to prove that thus defined function is of class $C_{K_{1}(\lambda)}^{1+\lambda}$ inside $A B C D$, we construct a family of curves connecting intervals $[B C]$ and $[D A]$ as follows. For $\sigma \in[0,1]$, let $z_{\sigma} \in[A B]$ and $w_{\sigma} \in[C D]$ be the points parametrized by $\sigma$, and $t_{\sigma} \in\left[z_{\sigma}, w_{\sigma}\right], t_{\sigma}=t_{z_{\sigma}, w_{\sigma}}$ as in (4.8). We parametrize each interval $\left[z_{\sigma}, t_{\sigma}\right]$ and $\left[t_{\sigma}, w_{\sigma}\right]$ by its normalized length such that $\tau\left(z_{\sigma}\right)=0, \tau\left(t_{\sigma}\right)=\frac{1}{2}, \tau\left(w_{\sigma}\right)=1$, and $\tau=$ const. gives us the second family of coordinate curves. Let $P=\left(\sigma_{1}, \tau_{1}\right)$ and $Q=\left(\sigma_{2}, \tau_{2}\right)$, and $R=\left(\sigma_{2}, \tau_{1}\right)$. There exist $C_{2}(\lambda), C_{3}(\lambda), K_{1}(\lambda)>0$ such that for $i=\sigma, \tau$

$$
\begin{aligned}
\left|F_{i}^{\prime}(Q)-F_{i}^{\prime}(P)\right| & \leq\left|F_{i}^{\prime}(Q)-F_{i}^{\prime}(R)\right|+\left|F_{i}^{\prime}(R)-F_{i}^{\prime}(P)\right| \\
& \left.\leq C_{2}(\lambda) d(Q, R)^{\lambda}+C_{3}(\lambda) d(R, P)^{\lambda}\right) \leq K_{1}(\lambda) d(P, Q)^{\lambda} .
\end{aligned}
$$

We use (4.8), (4.9) and (4.10) to obtain the second inequality. The last inequality follows from the regularity of the quadrangle $A B C D$ and the fact that the function $d^{\lambda}$ is concave down for any $\lambda, 0<\lambda \leq 1$.

Let us choose a finite cover of $X$ by cylinders $C_{\rho}\left(x_{i}\right), i=0, \ldots, N$ introduced in $\S 3$. We extend $F(x)$ by the formula

$$
F\left(\psi^{t} x\right)=\int_{0}^{t} f\left(\psi^{s} x\right) d s+F(x), \quad-\rho<t<\rho
$$

to a $C_{K_{1}(\lambda)}^{1+\lambda}$-function on each $C_{\rho}\left(x_{i}\right)$. Thus for $i=0, \ldots, N$ we obtain a function $F_{i}(x)$ defined on $C_{\rho}\left(x_{i}\right)$ and such that $F_{i}(x)=F(x)$ for $x \in \bar{\Lambda}_{i}$. Let $\left\{\lambda_{0}(x), \ldots, \lambda_{N}(x)\right\}$, $\sum_{i=0}^{N} \lambda_{i}(x)=1$ be a $C^{\infty}$ partition of unity corresponding to the cover $\left\{C_{\rho}\left(x_{i}\right)\right\}$, and $\bar{F}(x)=\sum_{i=0}^{N} \lambda_{i}(x) F_{i}(x)$. For

$$
x \in \bigcap_{k=1}^{M} C_{\rho}\left(x_{i_{k}}\right), \quad \mathscr{D} \bar{F}(x)=\sum_{k=1}^{M} \mathscr{D} \lambda_{i_{k}}(x) F_{i_{k}}(x)+\lambda_{i_{k}}(x) \mathscr{D} F_{i_{k}}(x) .
$$


By construction, on each $C_{\rho}\left(x_{i_{k}}\right)$ we have $\mathscr{D} F_{i_{k}}(x)=f(x)$ and $\lambda_{i}(x)=0$ if $i \neq i_{k}$. Thus

$$
\mathscr{D} \bar{F}(x)=\sum_{k=1}^{M} \mathscr{D} \lambda_{i_{k}}(x) F_{i_{k}}(x)+f(x),
$$

and therefore $\mathscr{D} \bar{F}(x)$ is of class $C_{K_{2}(\lambda)}^{1+\lambda}$ for some $K_{2}(\lambda)>0$.

Now we estimate the $C^{1}$-norm of $h(x)=\mathscr{D} \bar{F}(x)-f(x)$. Let $x \in \bigcap_{k=1}^{M} C_{p}\left(x_{i_{k}}\right)$. If $M=1, \bar{F}(x)=F_{i_{1}}(x)$, hence $\mathscr{D F}(x)=f(x)$ and $h(x)=0$ in some neighborhood of the point $x$. Therefore, in the open set $X \backslash \bigcup_{j \neq i_{1}} C_{\rho}\left(x_{j}\right)\|h(x)\|_{C^{1}}=0$. Suppose $M>1$. We notice that $\sum_{k=1}^{M} \mathscr{D} \lambda_{i_{k}}(x)=0$, and therefore $h(x)=\sum_{k=2}^{M} \mathscr{D} \lambda_{i_{k}}(x)\left(F_{i_{k}}(x)-F_{i_{1}}(x)\right)$. Since the functions $\lambda_{i_{k}}$ are of class $C^{\infty}$, we have in $\bigcap_{k=1}^{M} C_{\rho}\left(x_{i_{k}}\right)$

$$
\|h(x)\|_{C^{1}} \leq C_{4} \sum_{k=2}^{M}\left\|F_{i_{k}}(x)-F_{i_{1}}(x)\right\|_{C^{1}} .
$$

There exists a constant $C_{5}>0$ and two points $y \in \bar{\Lambda}_{i_{k}}, z \in \bar{\Lambda}_{i_{1}}$ such that $d(x, y) \leq \varepsilon$, $d(x, z) \leq \varepsilon, d(y, z) \leq C_{5} \varepsilon$. In the following estimate we use that the functions $F_{i_{k}}(x)$, $F_{i_{1}}(x)$ and $F(x)$ are of class $C^{1}$, and that $F_{i_{k}}(y)=F(y), F_{i_{1}}(z)=F(z)$.

$$
\begin{aligned}
\left|F_{i_{k}}(x)-F_{i_{1}}(x)\right| & \leq\left|F_{i_{k}}(x)-F_{i_{k}}(y)\right|+\left|F_{i_{1}}(z)-F_{i_{1}}(x)\right|+\left|F_{i_{k}}(y)-F_{i_{1}}(z)\right| \\
& =\left|F_{i_{k}}(x)-F_{i_{k}}(y)\right|+\left|F_{i_{1}}(z)-F_{i_{1}}(x)\right|+|F(y)-F(z)| \leq C_{6} \varepsilon .
\end{aligned}
$$

For $j=0, s, u$ the functions $\mathscr{D}_{j} F_{i_{k}}(x)$ and $\mathscr{D}_{j} F_{i_{1}}(x)$ satisfy a Hölder condition of order $\lambda$ and a constant $K_{1}(\lambda)$ for any $\lambda, 0<\lambda<1$. By construction (Lemma 4.1) we have $\mathscr{D}_{j} F_{i_{k}}(y)=k^{j}(y), \mathscr{D}_{j} F_{i_{1}}(z)=k^{j}(z)$ (see notations of $\S 2$ ), and using Theorem 2.1 we obtain the following estimate:

$$
\begin{aligned}
\left|\mathscr{D}_{j} F_{i_{k}}(x)-\mathscr{D}_{j} F_{i_{1}}(x)\right| \leq & \left|\mathscr{D}_{j} F_{i_{k}}(x)-\mathscr{D}_{j} F_{i_{k}}(y)\right| \\
& +\left|\mathscr{D}_{j} F_{i_{1}}(z)-\mathscr{D}_{j} F_{i_{1}}(x)\right|+\left|\mathscr{D}_{j} F_{i_{k}}(y)-\mathscr{D}_{j} F_{i_{1}}(z)\right| \\
\leq & C_{7}(\lambda) \varepsilon^{\lambda} .
\end{aligned}
$$

Thus, for some constant $K_{3}(\lambda)$ we have $\|h(x)\|_{C^{1}} \leq K_{3}(\lambda) \varepsilon^{\lambda}$, and the lemma follows.

Now we can finish the proof of Theorem 1.1. For any $\lambda, 0<\lambda<1$ there exists a constant $C_{8}(\lambda)>0$ such that for any $\varepsilon>0 \ln \varepsilon^{-1} \leq C_{8}(\lambda) \varepsilon^{\lambda-1}$. Given $T>0$, let

$$
\varepsilon=C^{1 /(3-\lambda)} C_{8}(\lambda)^{1 /(3-\lambda)} T^{-1 /(3-\lambda)},
$$

where $C$ is from Theorem 3.1. We apply Theorem 3.1 to construct an $\varepsilon$-dense piece of orbit $\mathcal{O}$ of length $C \ln \varepsilon^{-1} / \varepsilon^{2} \leq T$. Defining the function $F(x)$ on $\mathcal{O}$ by formula (4.1) and applying Lemmas 4.1 and 4.2 we obtain a function $h(x)$ with the following estimate on its $C^{1}$-norm:

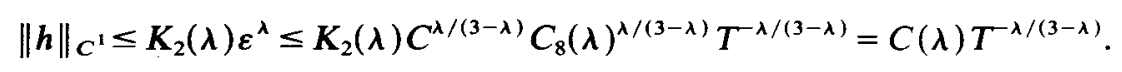

Acknowledgements. The main results of this paper were obtained in 1986-87 during my affiliation with UC San Diego as an NSF Postdoctoral Research Fellow. I am grateful to Audrey Terras and Harold Stark for their support and interest in this work. I acknowledge the support of SFB 170 'Geometrie und Analysis' in Göttingen, and appreciate the hospitality of the Stochastical Institute of the University of 
Göttingen during my visit in the summer of 1987 when this paper was mostly written. Finally, I would like to thank Paddy Patterson, Misha Brin and Don Zagier for valuable comments.

\section{REFERENCES}

[1] D. V. Anosov. Geodesic flows on closed Riemannian manifolds with negative curvature. Proc. Steklov Inst. of Math. 90 (1967).

[2] R. Bowen. Periodic orbits for hyperbolic flows. Amer. J. Math. 94 (1972), 1-30.

[3] V. Guillemin \& D. Kazhdan. On the cohomology of certain dynamical systems. Topology 19 (1980), 291-299.

[4] E. Hopf. Statistik der geodätischer Linien in Mannigfaltigkeiten negativer Krümmung. Ber. Verh. Sächs. Akad. Wiss. Leipzig 91 (1939), 261-304.

[5] E. Hopf. Statistik der Lösungen geodätischer Probleme vom unstabilen Typus. II. Math. Ann. 117 No. 4 (1940), 590-608.

[6] S. Hurder \& A. Katok. Differentiability, rigidity and Godbillon-Vey classes for Anosov flows, to appear, Publ. Math. IHES.

[7] A. N. Livčic. Some homology properties of $U$-systems. Mat. Zametki 10 (1971), 555-564; Math. Notes 10 (1971), 758-763.

[8] R. de la Llave, J. Marco \& R. Moriyon. Canonical perturbation theory of Anosov systems and regularity results for Livčic cohomology equation. Ann. of Math. 123 No. 3 (1986), 537-612. 